ALIANZAS ESTRATÉGICAS: STRATEGIG ALLIANGES: INSTRUMENTO DE NEGOCIACIÓN Y

\title{
DESARROLLO SOSTENIBLE
} MIRADO DESDE LA \section{INTERCULTURALIDAD}

FECHA DE RECEPCIÓN: 14 de enero FECHA DE APROBACIÓN:10 de marzo Pp. 102-117

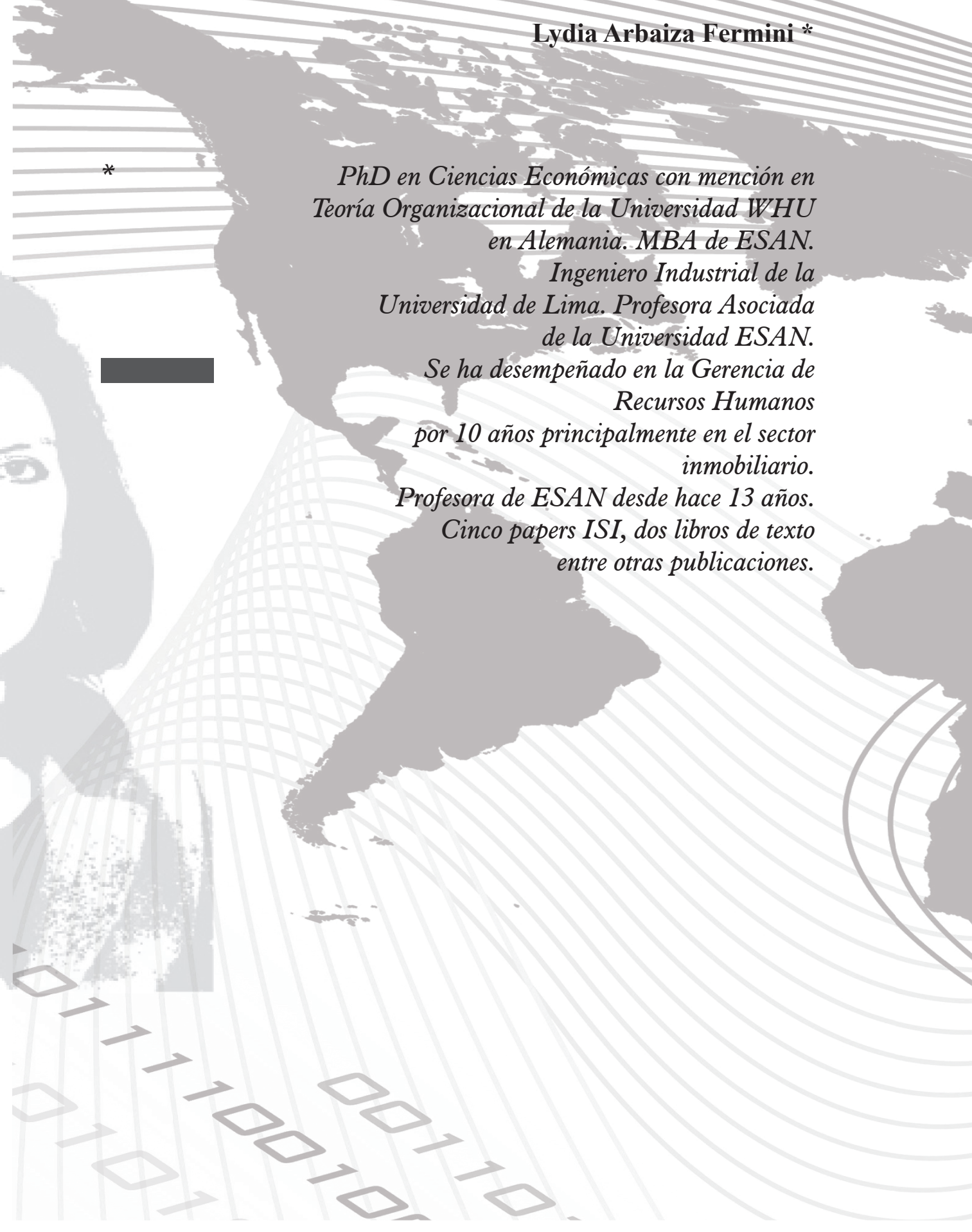




\section{RESUMEN}

La pobreza es un fenómeno que se manifiesta en la falta de acceso a la educación y servicios adecuados, la desnutrición, la falta de saneamiento y las desigualdades de género. Frente a ello, en países como el Perú se ha establecido un Fondo Social Minero, el cual tiene el potencial de convertirse en una herramienta estratégica poderosa para sentar las bases del desarrollo sostenible, reducción de la pobreza y para el desarrollo institucional. A su vez el Fondo ha traído como correlato la constitución de alianzas estratégicas, las que han ayudado a aumentar la productividad de los recursos, a abrir nuevos mercados y son un instrumento esencial de negociación entre las diferentes culturas implicadas en la inversión minera. En este artículo, se propone la aplicabilidad de un modelo de desarrollo basado por un lado en las tres estrategias en las que el Fondo Social Minero puede ser utilizado: desarrollo sostenible, reducción de la pobreza y desarrollo institucional; y de otro lado atravesado por un eje fundamental: la perspectiva intercultural. $\rightarrow$ Palabras claves

Interculturalidad Negociación

Alianzas estratégicas

Legitimidad

Fondos sociales mineros

Desarrollo sostenible

Desarrollo institucional

Reducción de la pobreza

\section{$\rightarrow \quad$ Key words}

Intercultural perspective Negotiation

Strategic alliances Legitimacy Social Mining Fund

Sustainable development

Institutional development

Poverty rate reduction process

In this paper, a new development model is presented based on the three strategies in which the Social Mining Fund can be applied, being sustainable development, poverty rate decrease and institutional development, and at the same time focused on a general axis-an intercultural perspective.

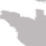
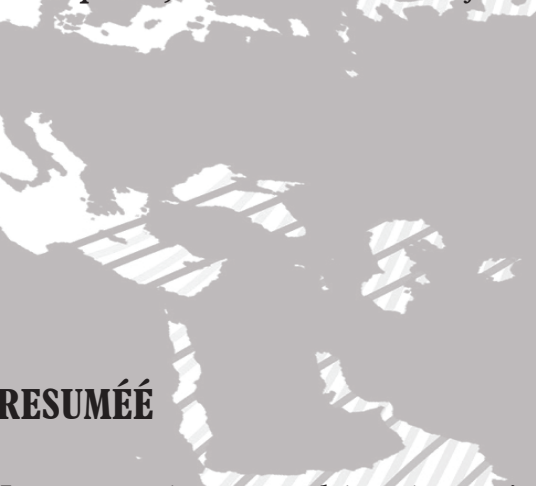

La pauvreté est un phénomène qui se manifeste par la difficulté d'accès à l'éducation, la malnutrition, l'absence d'assainissement et l'inégalité entre les sexes. Face à ces difficultés, un pays comme le Pérou a mis en place un Fond Social Minier qui a le potentiel pour devenir un puissant outil stratégique et ainsi créer les bases d'un développement durable, d'une réduction de la pauvreté permettant un développement institutionnel dans ce pays. Dans le même temps, le Fonds minier a eu pour corrélation la formation d'alliances stratégiques qui ont contribuées à l'accroissement de la productivité des ressources, à l'ouverture de nouveaux marchés et sont devenues un outil essentiel lors des négociations entre les différentes cultures impliquées dans l'investissement minier.

Dans cet article, l'application d'un modèle de développement est basè, d'une part, sur les trois stratégies grâce auxquelles le Fonds Social Minier peut être utilisé, entendez le développement durable, la réduction de la pauvreté et le développement institutionnel; et d'autre part, l'axe fondamental sous jacent de ce travail, la perspective interculturelle.

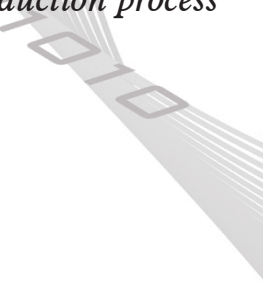




\section{INTRODUCción}

c desarrollados y menos desarrollados establecieron programas destinados a compensar el impacto negativo que tenía el ajuste estructural entre los pobres. Estos programas surgieron como una nueva estrategia de lucha contra la pobreza, realizando una intervención rápida, eficiente transfiriendo recursos y servicios a la población más necesitada. En ese contexto sirvieron para complementar las políticas sociales ya existentes. La mayor parte de estos programas se establecieron en África y América Latina bajo el nombre de Programas de Acción Social, Programas de Desarrollo Social, Fondos Sociales de Emergencia, Fondos de Solidaridad, o Fondos de Inversión Social. Hoy en día, estas regiones cuentan, con al menos 25 programas de este tipo (OIT, 2001). En el Perú, específicamente en el sector minero se han venido también estableciendo Fondos Sociales Mineros que han surgido en respuesta al llamado del gobierno de crear el Programa Minero de Solidaridad con el Pueblo, conocido como Aporte Voluntario (Horizonte Minero, 2009).

De aquí que, el objetivo central de este programa ha sido crear un fondo que es financiado por las empresa mineras que radican en el país. Este Fondo Social es una importante fuente de recursos para la inversión social en las zonas donde operan las mineras. En ese sentido, el Fondo Minero administrado por la Comisión Técnica de Coordinación se utiliza en proyectos de nutrición, educación, salud, desarrollo y fortalecimiento de capacidades de gestión pública, desarrollo en la promoción de cadenas productivas, infraestructura básica, obras de impacto local o regional, entre otros (Sociedad Nacional de Minería y Petróleo, 2010).

En base al reporte del MINEM de Marzo del 2010, son 39 las empresas que han firmado el Programa Minero de Solidaridad con el Pueblo, favoreciendo el desarrollo en 16 regiones del país, acumulando $\mathrm{S} / 1$ '388 millones aproximadamente para el Fondo Global. Según el Informe de la Sociedad nacional de Minería, Petróleo y Energía (2010) se está trabajando en 1'568 proyectos, de los cuales 886 ya han sido terminados y 187 están por culminar al $80 \%$. Asimismo, la región que reporta mayor número de proyectos es Ancash, Cuzco y Arequipa. Por otro lado, de los proyectos que se están realizando, el $47 \%$ corresponden al sector Nutrición, Educación y salud, seguidos de proyectos de Infraestructura. Por su parte, el rubro que mayor nivel de ejecución tiene es Fortalecimiento de capacidades, seguido de proyectos de promoción de cadenas productivas, salud, y educación (SNMPE, 2010).

Casos relevantes en el tema son el Fondo Minero Antamina (FMA), que ha invertido más de $\$ 80$ millones en mejorar la calidad de vida de la población, registrando logros como la disminución de la desnutrición crónica en 2.94\% entre los 25,000 niños atendidos en la región. Asimismo, el FMA busca fortalecer las relaciones entre el Estado y las organizaciones comunitarias favoreciendo la participación de los líderes comunales en los trabajos ejecutados (Horizonte Minero, 2009; Negocios Internacionales, 2009). Por tanto, el FMA se constituye como una iniciativa para el desarrollo sostenible del país (Negocios Internacionales, 2009).

Por su parte, la empresa Xstrata se caracteriza por el compromiso social que tiene con las comunidades. Tiene una comunicación con los involucrados en temas ambientales a través de charlas de sensibilización a los trabajadores. Así mismo, realiza mesas de diálogos en temas de tierras, medio ambiente y derechos humanos (Horizonte Minero, 2009, Proinversión, 2005). En ese sentido, El Fondo Social beneficia a más de 83 mil habitantes en 20 distritos y 150 comunidades campesinas, ejecutando proyectos de agua potable, electrificación, salud, educación, agricultura, construcción, etc. (Minería, 2009). 
Igualmente, la empresa minera Yanacocha, también ha destinado recursos a la ejecución de obras relacionada con la nutrición de menores de 0 a 5 años y madres gestantes, a la educación primaria, a la salud, a la infraestructura básica (agua, luz), al fortalecimiento de capacidades de la gestión pública. En la actualidad, el aporte de la minera Yanacocha al Fondo Social asciende a \$49.6 millones entre el 2007 y 2009 (CEDEPAS, 2009).

Si bien se observa un notable incremento de políticas destinadas a mejorar la calidad de vida de la población, nos preguntamos ¿por qué existe todavía un gran sector de la población que no ha sido beneficiada de estos recursos que aporta el sector minero? Y ¿por qué aun existen conflictos entre las comunidades y las mineras?

Según lo señalado por el Banco Interamericano de Desarrollo (2003) si bien América Latina y el Caribe han logrado reducir la pobreza en los años noventa, la pobreza aun afecta a más de un tercio de la población de la región.
Esta pobreza es mayor en zonas rurales, (59.1\%) y está asociada al bajo nivel educativo y al pertenecer a grupos indígenas o africanos. Posiblemente, en el Perú, estos resultados nos hacen pensar en cómo se está gestionando desde los gobiernos locales los recursos brindados por el Fondo Minero. Así mismo, no se debe de perder de vista que existe un importante sector de las comunidades rurales ubicadas en zonas mineras que tienen una percepción negativa del significado de invertir en la minería (Guzmán, 2003). Esta situación ha hecho que se generen en todo el país conflictos sociales. Estas dificultades han surgido en su mayoría por la preocupación por el medio ambiente (Tanaka \& Huber, 2007). El presente trabajo tiene por finalidad plantear un modelo en el que el Fondo Minero funcione como estrategia para temas de Desarrollo Sostenible, Reducción de la pobreza y Desarrollo Institucional siempre y cuando se tome en cuenta la perspectiva de la interculturalidad. Asimismo, este Fondo minero ha traído como correlato la formación de alianzas entre instituciones según las necesidades que persiguen.

\section{(4) \\ 2. LEGITIMIDAD DE LAS ALIANZAS ESTRATÉGICAS}

ulati (1998) se refiere al término alianzas estratégicas como las relaciones voluntarias entre organizaciones en uno o varias esferas de actividad en el que ambas partes regulan su comportamiento futuro a través de la mutua tolerancia. Así mismo, se le conoce en un sentido más básico como las iniciativas del sector público, privado y del sector sin fines de lucro de contribuir con recursos financieros, humanos, técnicos e intangibles para lograr los objetivos propuestos (Casado, 2007; Das, \& Teng, 2000; Fiszbein \& Lowden, 1999; Montes \& Sabater, 2002).

Otros autores las definen como los acuerdos de colaboración en el que las agencias del sector público entablan relaciones contractuales a largo plazo con entidades del sector privado con el fin de que estas últimas construyan o gestionen la infraestructura pública, o provean de servicios a la comunidad (Zarco-Jasso, 2005).

Las alianzas han tenido un notable incremento en los últimos años debido principalmente a que muchas empresas, incluso las más poderosas han percibido que sus recursos humanos, tecnológicos, y financieros son limitados. Es por ello que se han empezado a aliar con otras instituciones para de esta manera seguir compitiendo y desarrollando una estrategia de colaboración. (Inforesources, 2005; Renart, 2008). En ese sentido, se considera que gracias a las alianzas la productividad de los recursos es potenciada, permiten el ingreso a nuevos mercados, y es posible el intercambio en temas estratégicos y de competencias o habilidades (Dyer, Kale \& Singh, 
2001; Fiszbein \& Lowden, 1999; García-Canal et al., 2004; Ireland, Hitt \& Vaidyanath, 2002). Se considera que para lograr el éxito esperado, las alianzas deben cumplir con ciertos principios que regirán durante todo el proceso (Montes \& Sabater, 2002). En primer lugar, se debe tener el objetivo de que cada socio o aliado "gane"; Lorange, Roos \& Simcic (1992) sostienen que cada una de las partes tiene necesariamente que verse favorecida. De otro lado, debe de haber un fuerte compromiso, es decir, debe de establecerse un lazo de confianza y un sentido de compromiso con la misma alianza (Gulati, Khanna \& Nohria, 1994). Asimismo, la alianza implica a su vez un proceso de flexibilidad, de transparencia en el que se da y se recibe, y se aprende lo que los socios pueden brindar y lo que ellos esperan recibir (Montes \& Sabater, 2002). De la misma manera, en el establecimiento de la alianza resulta fundamental mantener una comunicación clara para llegar a un buen entendimiento entre sus partes (Reardon \& Spekman, 1994). Finalmente, es imperativo crear un ambiente de equidad y justicia, es decir, que cada socio perciba que los demás aliados van a cumplir con lo prometido y que la alianza tendrá un fin productivo. En ese sentido, las relaciones interpersonales entre aliados tendrán mayor confianza (Smith \& Van de Ven, 1994).

Si bien la literatura muestra un importante interés sobre las ventajas del establecimiento de alianzas no se ha prestado la suficiente atención a la necesidad de legitimarlas o darles credibilidad. De ahí que, para su establecimiento, la legitimidad es esencialmente relevante y se refiere a la percepción que tiene una institución sobre otra (Kumar $\&$ Das, 2007). En otras palabras se refiere al proceso de validación social en el que se reconoce una competencia o cualidad distintiva de la institución (Dacin, Oliver \& Roy, 2006). Según Suchman (1995), en el concepto de legitimidad está implícita la percepción que las acciones de una entidad son apropiadas, adecuadas o deseables dentro del sistema de normas, valores o creencias. En ese sentido, la legitimidad corresponde a qué tanto las actividades de una institución son compatibles con las normas sociales, valores, con las expectativas de la organización y con el entorno social (Dacin et al., 2006).

Los estudios realizados sobre el tema muestran diferentes clasificaciones o tipologías sobre los tipos de legitimidad de las alianzas las cuales han sido revisadas por Kumar \&. Das (2007). Se propone un tipo de legitimidad interna, y otra externa; una legitimidad sociopolítica y cognitiva; u otra clasificación desarrollada por Suchman (1995) que señalan la legitimidad pragmática, la moral y la cognitiva. En esa línea, (Dacin et al.2006) han propuesto otra tipología que está basada en la percepción de la propia institución. En este modelo se analizan cinco ejes de legitimidad. Los autores señalan que al realizar un examen de legitimación de las alianzas se está potenciando el rendimiento económico y la competitividad de las instituciones.

La distinción que realizan está en función de las características de los conductores denecesidad (need drivers), que -al ser tanto del entorno como de la misma organizaciónse conjugan en una determinada preponderancia. En otras palabras, de la conjunción de need drivers de la empresa y del entorno, la alianza estratégica establecida estará más o menos orientada a alguna de las clases de legitimidad planteadas.

En primer lugar, se menciona una legitimidad de mercado, definida como aquella en la cual la organización busca una mayor participación y presencia en el mercado; lo que la motiva es una mayor legitimidad en un mercado geográfico o de producto. Esta clase de legitimidad responde a características del entorno delineadas por el apoyo del gobierno o de un respaldo para el acceso al mercado. De otro lado, los need drivers de la organización, en este caso, referirían tanto a una experiencia o mayor reputación en ese sector del mercado, así como una performance anterior en este. Por tal motivo, la fuente de legitimidad para la organización que se embarca en una alianza estratégica con esta preponderancia proviene de la legitimidad que ya tenga el socio en el mercado. De otro lado, esta legitimidad busca proyectarse hacia los gobiernos, proveedores y clientes. En segundo lugar, la legitimidad relacional es aquella que enfatiza sobre el valor de asociarse. Por eso, la fuente de esta clase de legitimidad proviene precisamente de la relación establecida con el socio, al tiempo que el foco de este tipo de alianzas reposa en los lazos potenciales que pueden establecerse. Esta clase de legitimidad está dictada, del lado de la empresa, por need drivers que se orientan a un historial de alianzas en la organización, así como la importancia de las alianzas en el negocio. En razón de factores del entorno, en cambio, orientan este tipo de alianzas tanto la competencia por socios atractivos como la necesidad de sedimentar lazos a futuro. Por otra parte, la legitimidad social, refiere a la consonancia de la alianza estratégica establecida por la organización con las expectativas y reglas sociales, una suerte de búsqueda de incrementar una mejor imagen social (el aspecto "socialmente responsable" descansa también en esto). De 
allí que la fuente de esta clase de legitimidad esté del lado de la imagen social que tenga el socio. Por eso, también, esta legitimidad se focaliza tanto en las comunidades que perciben la actividad de la organización como en los grupos de interés público. Así, el factor del entorno resulta vital en tanto motivador de este tipo de alianzas, ya sea porque precisamente el entorno social es el que monitorea la imagen social de la empresa o porque necesita proyectar una imagen socialmente responsable para sobrevivir. Del lado de la organización, en cambio, buscar este tipo de alianzas suele estar motivado por proyectar una mayor visibilidad de las actividades o por tener un mayor impacto social como firma al igual que mejorar su imagen.

Otra legitimidad planteada por Dacin es la legitimidad de inversión. Tal como señala su nombre, este tipo de alianza se orienta a mejorar la legitimidad de negocios de la empresa, de la actividad misma. En otras palabras, al ser la fuente de la legitimidad el soporte y aporte del socio para la actividad de negocio, $\mathrm{y}$ al estar focalizada a tener efecto sobre inversionistas y directores de otras compañías, esta legitimidad refuerza "la imagen como negocio" de una organización. Por eso, lo que motivaría una alianza como esta, desde la organización, sería tanto una adopción de determinada actividad en pos del negocio, por parte de la organización, como la necesidad por la inversión o el aumento de recursos para realizar las mismas actividades. Por parte del entorno, la motivación de esta clase de alianzas se determinaría tanto por la necesidad de poner en manos del socio determinada actividad de un negocio, como por la promoción de entidades legitimadas.

Finalmente, la legitimidad de alianza, cuya fuente está en el nivel de certitud de determinada alianza. Es decir, que la alianza se legitima en tanto ha sido una decisión oportuna, una "jugada acertada". Esta clase de alianza legitima a las alianzas en sí. No a una determinada alianza, sino a la actividad de realizar alianzas estratégicas, y lo hacen por lo acertadas que han resultado. De allí que el foco de esta clase de legitimidad sean otras organizaciones en general. El entorno motivaría esta clase de alianzas en tanto el mercado las hace factibles, consecuentes. Del lado de la empresa, una alianza de este tipo se determina en tanto busca el éxito de la firma o la aceptación en el mercado mismo. Las investigaciones muestran que el satisfacer las necesidades de legitimización ha traído como resultado que las organizaciones sean beneficiarias de diversos recursos estratégicos como mejor reputación, acceso a otros mercados, el tener una imagen positiva frente a ciertos grupos objetivo. De ahí que, las alianzas hayan mejorado su desempeño (Baum \& Oliver, 1991; Larson, 1992).

\section{6 \\ 3. INTERCULTURALIDAD EN LA GESTIÓN}

a interculturalidad se relaciona estrechamente con el intercambio entre dos o más culturas de manera tal que se respete a cada grupo o cultura. Deben primar los conceptos de integración y tolerancia en la convivencia intercultural. Los principios de la interculturalidad apuntan a la tolerancia de la diversidad. En este proceso de habla de la interacción entre todo aquel conjunto de formas, modelos o patrones, explícitos o implícitos, a través de los cuales una sociedad regula el comportamiento de las personas que la conforman. La cultura brinda a los seres humanos la capacidad de reflexión sobre él mismo. La cultura hace de los hombres seres racionales, humanos, críticos y éticamente comprometidos. Es un

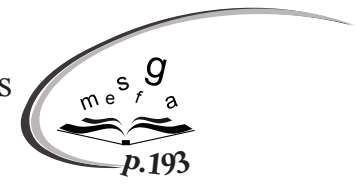
medio de expresión de nuestras propias metas, significados. (Unesco, 1982). 
Ahora bien, la cultura es adquirida a través de la transmisión de valores creencias y va a determinar el comportamiento de las personas y de grupos. Hall plantea que la cultura se puede aprender a través del aprendizaje formal, el informal y el técnico. El aprendizaje formal es el que se relaciona con la trasmisión de padres a hijos. El informal, el que se refiere al que es aprendido a través de modelos. Finalmente el aprendizaje técnico se genera a través de la interacción entre personas que pretenden comentar conceptos ya aprendidos, o llegar a acuerdos (Maldonado, 2006). De otro lado, Maldonado (2006) hace una revisión sobre lo propuesto por el investigador Hofstede (1997) quien propone que los valores y creencias son adquiridos de una manera inconsciente. De modo que, la persona empieza a construir un mapa mental que va a regir sus nuevas experiencias.

Ahora, se dice que las relaciones entre culturas entrarían en el campo del aprendizaje técnico, pues es en este momento donde se pueden generar nuevas concepciones, visiones, valores, etc. Sin embargo, es aquí donde pueden surgir precisamente las diferencias entre culturas pues cada persona o grupo tiene una programación mental que puede chocar con otro diferente a la propia. Es este elemento el que está implicado en la negociación internacional. Es muy posible que surja desinformación, prejuicios, incertidumbre, frustración y malestar al producirse el contacto entre culturas.

En ese sentido, hablamos de interculturalidad como el reconocimiento de las dificultades que surgen entre culturas diferentes planteando la posibilidad de compartir valores comunes siendo el resultado de un proceso de convivencia, diálogo y disponibilidad para la comprensión mutua. Ahora es posible determinar ciertas dimensiones que según Hofsede resultan esenciales en el momento de la comprensión intercultural. Estas son: la distancia y distribución del poder, el individualismo y colectivismo; el manejo de la incertidumbre, la masculinidad y femineidad; la concepción de largo y corto plazo (Maldonado, 2006).

Ahora, desde la administración empresarial, los elementos de interculturalidad están muy presentes hoy en día pues ya no existe ni un solo modelo de funcionamiento, ni de ambiente económico y social que pueda quedarse aislado del resto del mundo. La empresa y los que en ella trabajan se ven frecuentemente enfrentados a diferentes puntos de vista y a diversas dificultades. Así mismo, las grandes empresas transnacionales tienen personal en su mayoría trabajando en el extranjero, así como una gran variedad de clientes. Finalmente, están las empresas que cuentan con un conjunto de empleados cada vez más diversificado. En ese sentido, la administración intercultural definida como: "el estudio de la conducta organizacional al interior de países y culturas; la comprensión para mejorar la interacción entre los trabajadores, los clientes, los proveedores y los socios de alianzas de diferentes países y culturas" resulta una visión más completa del estudio de la administración misma pues logra tener un alcance multicultural (Adler,1991).

Además interviene en las actividades de negociación como transacciones internacionales o en las actividades de cooperación como en alianzas. Igualmente esta gestión toma en cuenta los grupos de culturas diferentes y que deben cooperar dentro de la misma organización.

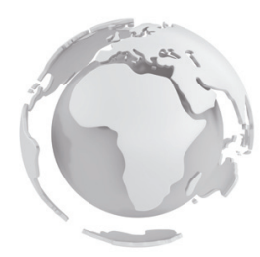




\section{ALIANZAS ESTRATÉGICAS: UN MODELO PARA EL DESARROLLO MIRADO DESDE LA PERSPECTIVA DE LA INTERCULTURALIDAD}

a pobreza es un fenómeno multidimensional que se manifiesta en una variedad de maneras, incluyendo la falta de acceso a la educación y servicios adecuados, la desnutrición, la falta de saneamiento y las desigualdades de género (Cichon et al., 2003). Este fenómeno constituye una amenaza que atenta el bienestar de los seres humanos, por tanto, su reducción sigue siendo principalmente un tema nacional y social de las políticas económicas (ProInversión, 2005).

Frente a ello, la distribución del Fondo Social Minero tiene el potencial de convertirse en una herramienta estratégica poderosa para sentar las bases del desarrollo sostenible, para la reducción de la pobreza y para el desarrollo institucional.

En cuanto al desarrollo sostenible, los fondos mineros pueden y deben considerar la protección del medio ambiente. Esto debe hacerse de una manera que garantice la ejecución presupuestaria eficiente y reducir el impacto ecológico en las regiones en las que proyectos se realizan. La ley que regula el plan de cerrar las minas y el reciente

Resolución Ministerial (515-2006-MEM/ DM) que garantiza el "plan para cerrar las minas con los fideicomisos para la inversión en los bosques" representan una oportunidad que no debe pasarse por alto.

La persona jurídica a cargo del Fondo Social aprueba el seguimiento de los proyectos. Por lo tanto, será necesario utilizar un enfoque de desarrollo integral de gestión de toda la vida útil de un proyecto. Las personas responsables del proyecto, o los ejecutores correspondientes, deben hacer una lista y analizar los esfuerzos o los elementos que impulsan o facilitan un proyecto. Esta evaluación también debe mirar todas las restricciones que se han planteado que impiden el funcionamiento del proyecto de forma adecuada (Arbaiza, 2008). Por ejemplo, el proyecto Las Bambas mineras contempla un enfoque sostenible del desarrollo que se basa en el principio de la inversión minera que es ecológicamente responsable y se centra en el desarrollo social. El desarrollo sostenible en el área de influencia es indispensable para el éxito del proyecto minero. Si la población percibe los beneficios de la inversión minera y estos beneficios perduran en el tiempo, promoverá la inversión privada. Esto generará un círculo virtuoso de prosperidad y bienestar en el futuro (PROINVERSIÓN, 2005).

Entre los proyectos de desarrollo sostenible se incluyen la construcción y mantenimiento de caminos rurales y la conexión de otras vías para que los productores rurales a los mercados establecidos, lo que reduce los costos de transporte. Esta disminución permitirá a los campesinos a vender sus productos a mejores precios y traerá nuevos productores en el mercado. De esta manera, los mecanismos de comercialización será diversificada, la reducción del poder de los intermediarios y facilitar las migraciones temporales y el acceso de las familias rurales cada vez más a los servicios básicos como la educación (en particular, secundaria y superior) y salud (Francke \& Iguiñiz, 2006).

La estrategia de desarrollo sostenible se asocia con el concepto de Responsabilidad Social Corporativa, que de acuerdo con Penny y mencionada por Arbaiza (2008) señala que las empresas deben hacer un compromiso con la comunidad a través de sus acciones. Deben proporcionar apoyo a los pobres, a las comunidades remotas; y tratar de construir un futuro mejor; así mismo, contribuir a la salud y la protección del

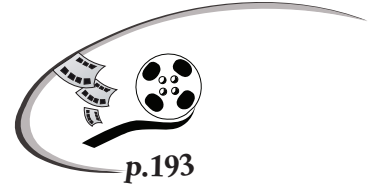
medio ambiente, y garantizar el desarrollo de la sociedad por medio de una serie de actividades, tales que pueden ofrecer oportunidades a los menos afortunados. En este sentido, la búsqueda de aumentar la cantidad y / o calidad del capital humano, a través de la inversión en recursos humanos (dentro y fuera de la empresa) cumple con estos requisitos. Parte de esto se puede lograr con el Fondo Social. 
Por otro lado, con respecto a la reducción de la pobreza, el Fondo Social ha jugado un papel importante en el apoyo a la mejora de la estructura social y económica y en los procesos para formar capital humano (Jorgensen \& Domelen, 1999). En ese sentido, estos fondos facilitan la ejecución descentralizada de las obras de pequeños proyectos en miles de comunidades pobres, impulsada por el hecho de que los recursos correspondientes se basan en la demanda, así como un enfoque participativo y constituyen una alternativa de bajo costo que rápidamente se desembolsa (Tendler, 2000). Expertos en la materia coinciden en que uno de los principales impedimentos a la ejecución del gasto se relaciona con la capacidad de la mala gestión en el nivel de los gobiernos regionales y los municipios beneficiarios.

Ahora bien, la experiencia acumulada demuestra que estos fondos constituyen mecanismos eficaces para controlar los flujos de financiamiento para proyectos locales y aliviar la pobreza a pesar de que ninguno de estos fondos ha sido capaz de erradicar por completo este flagelo en el área de intervención. Como regla general, los fondos que han utilizado diseños más objetivos han tenido más éxito. También es digno de mención que los fondos han tenido un impacto transitorio ya que en varios casos, el énfasis ha sido en la creación de puestos de trabajo temporal, y no en el promover la actividad productiva, la creación de empleo permanente y la generación de ingresos entre los pobres.

Los fondos sociales son claramente un complemento y no un sustituto de las políticas del sector y son incapaces de satisfacer todas las necesidades de las comunidades pobres. Estos fondos deben trabajar en conjunto con las organizaciones no gubernamentales, comunidades y otros agentes de desarrollo para contribuir inversiones que son capaces de alcanzar el objetivo más grande o aliviar la pobreza.

De otro lado, en cuando al desarrollo institucional, los Fondos sociales han demostrado que son eficaces e innovadoras organizaciones que utilizan procedimientos transparentes para canalizar recursos de las comunidades. Un porcentaje significativo de esta eficacia se debe a su carácter autónomo, que les da la libertad en términos de contratación y procedimientos de operación (Banco Mundial, 2002). Los objetivos de desarrollo institucional de los proyectos de fondos sociales se han desplazado desde el ámbito del gobierno central hacia los gobiernos locales y las comunidades. El impacto a nivel local ha sido positivo, varían en función de la naturaleza social de los fondos sociales, del compromiso y el grado de responsabilidad delegada a los gobiernos locales y las comunidades. (Banco Mundial, 2002).

Los fondos sociales tienden a ser más participativos que otros proyectos de desarrollo, pero tienen el potencial de mejorar aún más. Estos fondos, que pueden variar considerablemente, el trabajo para estimular las comunidades de la confianza en el sector público y generar el capital social. (Jorgensen, 1999).

Los fondos sociales tienden a tener dos objetivos: aumentar el pobre acceso a los servicios e infraestructuras locales y la autonomía de las comunidades mediante su participación en la selección, ejecución, operación y mantenimiento de proyectos de desarrollo. Los fondos sociales han desarrollado mecanismos para canalizar directamente a los grupos en la comunidad. Se pueden asumir responsabilidades para este tipo de actividad, que incluyen la organización de la comunidad, las contribuciones de trabajo, adquisición de bienes y servicios y otras tareas de

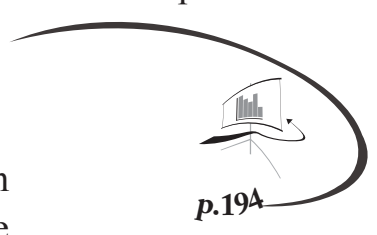
gestión de proyectos. Estos fondos han ayudado a mejorar los servicios básicos. (Parker, 2000).

Con el fin de afinar las comunidades institucionales de desarrollo, es fundamental tener un conocimiento preciso de las características de la población. Esto constituye un elemento esencial en la gestión moderna de las instituciones como los municipios, cuya principal función consiste en prestar servicios que mejoren las comunidades el nivel de vida. Esta información será útil para satisfacer las necesidades de los residentes y proporcionar las bases para generar un sistema de información para el desarrollo social, que es un componente fundamental en la gestión municipal (Arbaiza, 2008). Esto permitirá a los municipios contribuir a las reuniones con los miembros del fondo social y de conocimiento para dar prioridad a los proyectos.

Como se ha señalado anteriormente, frente al deterioro socio ambiental percibido mundialmente se empiezan a manifestar diferentes iniciativas para contrarrestar sus consecuencias. Por un lado, en el año 2000 se aprueba en 189 países los objetivos del desarrollo del milenio, cuya 
propuesta se refiere a actividades y metas en favor de la reducción de la pobreza y mortalidad infantil, promoción de la igualdad, garantizar la sostenibilidad del medio ambiente entre otros (Naciones Unidas, 2000). Esto ha traído como resultado que, se empiecen a establecer Alianzas entre los diferentes sectores de la sociedad, (sector público, sector privado, organizaciones de la sociedad civil) quienes se han propuesto desarrollar diferentes proyecto o programas de acción a favor de los objetivos del milenio.

La experiencia ha mostrado que las alianzas públicoprivadas garantizan mayores inversiones en países menos desarrollados, fomentando el avance de las comunidades más pobres y el respeto de los derechos humanos. Casos internacionales como la alianza para promover la educación en Jordania (JEI), la alianza global para vaccines and immunization (GAVI) entre el sector público y privado que incluye a organizaciones internacionales como UNICEF, OMS, Banco Mundial, Gobiernos de Canadá, Dinamarca, Francia, Irlanda, Luxemburgo. Holanda, Noruega, Suecia y Gran Bretaña, y ONG y centro de investigación (GAVI), Global Partnership to stop tuberculosis (STOPTB), Poverty-Environment Partnership (PEP), Global Water Partnership, growing Sustanaible Business del PNUD, etc. han demostrado tener un nuevo modelo de trabajo, pues aportan competencias estratégicas, soluciones innovadoras, son una fuente adicional de recursos financieros, técnicos, de conocimientos para los gobiernos, así mismo brindan apoyo técnico y financiación adicional a la socie- dad civil como acceder a nuevos contratos y desarrollar nuevos productos o servicios colaborando con el sector privado (Casado, 2007).

Sin embargo, al establecerse una alianza es preciso fijar objetivos comunes, beneficios de cada socio, establecer una distribución equitativa de poder en la toma de decisiones. De ahí que muchas veces, se generen ciertos obstáculos en el momento de celebrarse la alianza. Al respecto, Tennyson (2003) refiere que frecuentemente existe una actitud escéptica de parte de un público general, limitaciones de las instituciones o de las personas que dirigen la alianza o características negativas percibidas de cada sector. Igualmente, se piensa que aun no existe una comprensión clara sobre el concepto de alianza, sobre cuándo es adecuado establecerla o las motivaciones para su aplicación y sobre cómo se gestiona o evalúa (Naciones Unidas, 2005). Por lo tanto, sólo si las alianzas muestran una adecuada gestión sin perder de vista el objetivo principal de su establecimiento tienen la capacidad para convertirse en una herramienta relevante para generar desarrollo social (Casado, 2007).

En ese sentido, se puede observar que el Fondo Social Minero se distribuye para el Desarrollo Sostenible, la Reducción de la Pobreza y para el desarrollo Institucional. En la estrategia de Desarrollo Sostenible (Figura 1), se definen los proyectos o programas ecológicos, económicos y sociales cuyo beneficio es la armonía de las comunidades con el entorno.

\section{Figura 1. Estrategia de desarrollo sostenible}

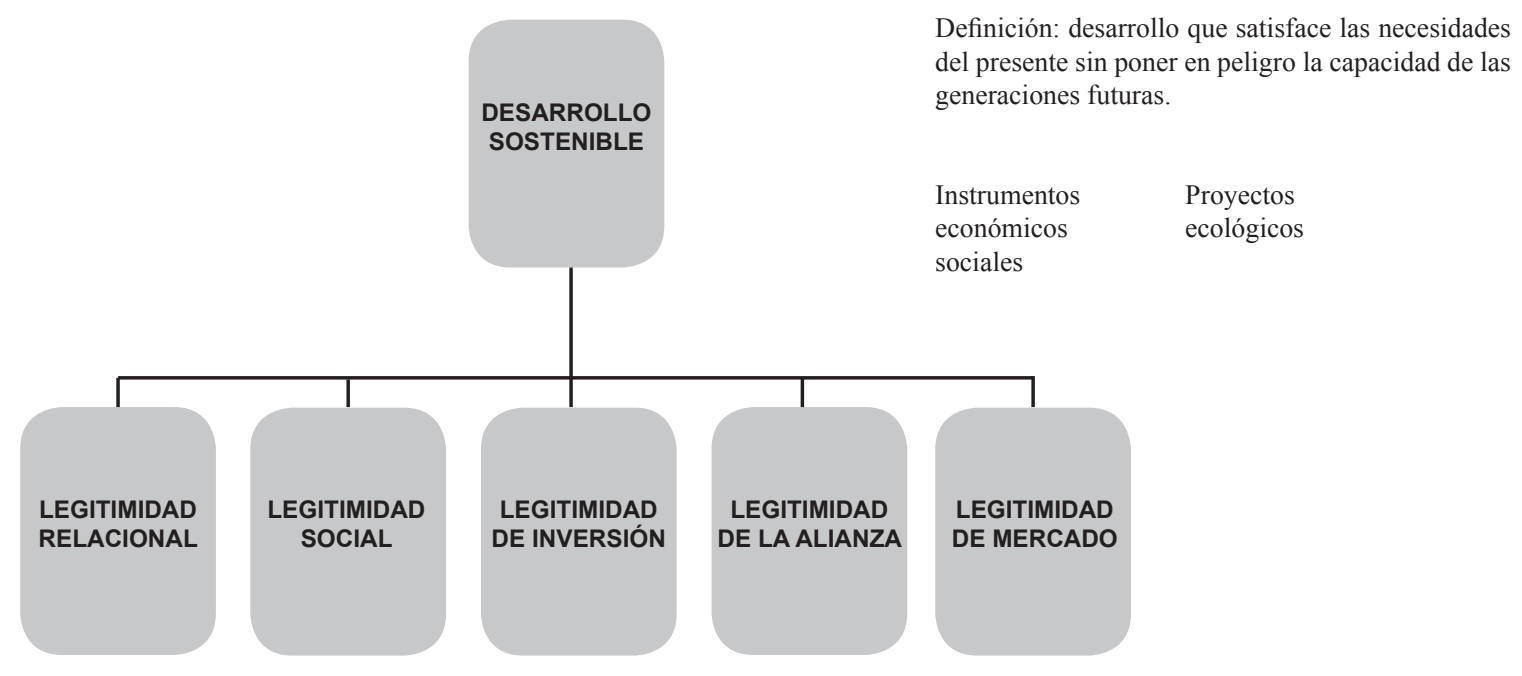

Fuente. Autora. 
Así mismo, el gobierno, con el plan de desarrollo estatal junto con otras instituciones de la sociedad civil y empresas privadas son los actores en esta estrategia. En ese sentido, el establecer proyectos de desarrollo sostenible favorece la formación de alianzas estratégicas entre los actores mencionados anteriormente que van a tener cada cual una o varias necesidades de aliarse para satisfacer una determinada legitimación. Es por ello que esta estrategia a su vez se complementa con la división en cinco perspectivas de legitimidad para las organizaciones.

En el primer tipo de legitimidad (de mercado) la institución aspira a una mayor presencia en el mercado pues requiere de una mayor reputación. Así, la fuente de legitimación proviene de la legitimidad que ya tenga el socio en el mercado. Finalmente, los beneficios son la entrada al mercado o continuar la existencia en ese mercado y su público objetivo es el gobierno, los clientes. Aquí resulta conveniente mencionar por ejemplo casos como la Minera Barrick Misquichilca que destina parte de sus fondos a proyectos de desarrollo sostenible beneficiando a pobladores de Cushuro, Chuyugal y Corral Grande en la región de La Libertad; paralelamente es parte de alianzas estratégicas en las que busca continuar en el mercado internacional obteniendo así una legitimidad de mercado. De otro lado, logra legitimidad social pues las comunidades locales la perciben como una empresa de responsabilidad social.

En el segundo tipo de legitimidad (relacional), se valora el asociarse, de ahí que la fuente de legitimidad sea la relación establecida con el socio y el objetivo son los lazos potenciales en el futuro. Esta legitimidad está orientada desde la empresa por need drivers orientados al historial de alianzas. Desde el entorno, se orienta hacia la competencia por socios atractivos. La motivación es aumentar la credibilidad o validez como un buen socio. Casos como la alianza entre la empresa norteramericana Newmont Mining Corporation, el grupo minero nacional Buenaventura y la International Finance han dado cuenta de la valoración del lazo. De ahí que, por ejemplo en grupo Buenaventura es uno de los grupos mineros más fuertes del Perú y tiene una trayectoria de historia de alianzas con otras entidades. A su vez, esta alianza que trabaja en Perú a través de Yanacocha, tiene a su vez legitimidad de mercado al mantener una reputación y aprobación del gobierno y clientes. Asimismo, logra legitimidad social frente a las comunidades locales pues promueve actividades de responsabilidad social. Por lo tanto, una alianza entre Newmont y Buenaventura, brinda beneficios a ambas empresas. Newmont tiene acceso a un yacimiento con costos de producción muy bajos (US\$ 104 por onza) que le permite contrapesar los costos de sus operaciones en Estados Unidos (US\$ 210), en Europa del Este (US\$ 225) y en Indonesia (US\$224). Yanacocha es, después de Carlin Trend, la segunda más grande unidad operativa de Newmont con 811.400 onzas de oro -de las cuales le corresponden 416.654 onzas. Es decir, Yanacocha le permite a Newmont mantener su posición de liderazgo en la industria del oro. De otro lado, una alianza estratégica con una empresa peruana diminuye el riesgo de la inversión de Newmont y le permite acceder a ámbitos del poder político peruano. Finalmente, Yanacocha le permite a Newmont aplicar su expertise geológico y minero minimizando los riesgos inherentes a toda operación minera.

Luego, desde la visión de Buenaventura, este grupo se ha convertido en el primero que desarrolla un proyecto de gran minería. De otro lado, Yanacocha representa aprendizaje y transferencia tecnológica en el tratamiento hidrometalúrgico de minerales, así como la expansión de las actividades de otras empresas del grupo.

En tercer lugar, la legitimidad social, como ya se ha revisando anteriormente, implica una armonía entre la institución u organización con las expectativas y normas sociales. En ese sentido, es esencial la imagen social que tenga el socio, frente a las comunidades que perciben la actividad de la organización. En este tipo de legitimidad el entorno juega un rol clave en la medida que monitorea la imagen social de la empresa. Del lado de la organización, se busca proyectar una mejor imagen a través de mostrar las actividades realizadas. Organizaciones que forman parte de este tipo de alianzas con minera Yanacocha, Barrick, Xstrata, Antamina, Poderosa, Marcobre, etc. Que a su vez, cada una de ellas mantiene otros tipos de credibilidad y paralelamente se ubican como fomentadores de la estrategia de desarrollo sostenible.

Por otro lado, la tabla muestra la legitimidad de inversión que se orienta a favorecer la credibilidad de negocios de la actividad de la empresa. Los socios tienen la necesidad de inversión o el aumento de recursos. Esta legitimidad está basada en el apoyo del socio y la confianza en la actividad comercial.

Finalmente existe una legitimidad del propio acto de establecer una alianza estratégica. Su foco de acción son 
las organizaciones en general. El beneficio es la aceptación social de la alianza.

De otro lado, el figura 2 muestra la estrategia de Reducción de la pobreza, en la que se desarrollan proyectos de responsabilidad social para reducir los conflictos sociales y mejorar la calidad de vida de las comunidades ubicadas en zonas mineras. Esta manera en la que se distribuye el Fondo Minero desencadena en alianzas entre las mineras, el estado, los gobiernos locales y ONG. Paralelamente estas alianzas satisfacen diversas necesidades de legitimidad tal y como se plantean en la estrategia de Desarrollo Sostenible.

\section{Figura 2. Estrategia de reducción de la pobreza}

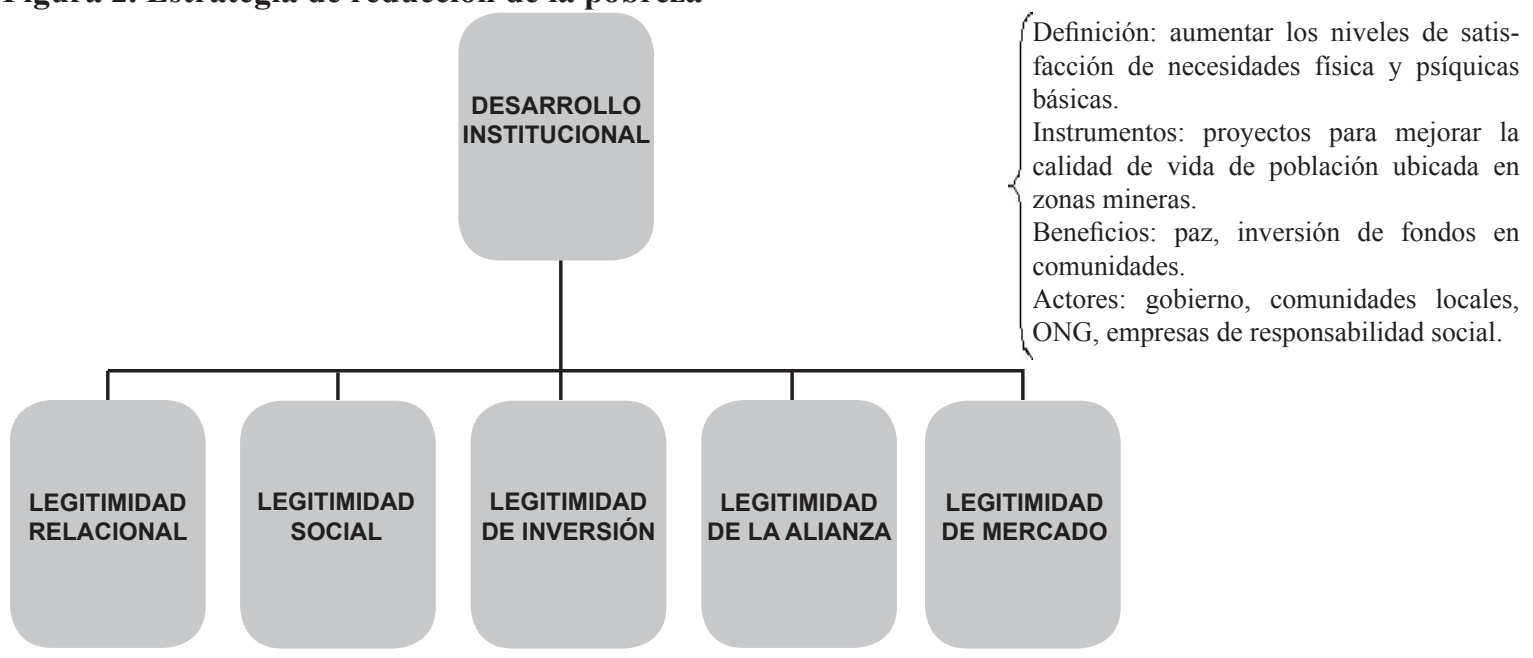

Fuente. Autora.

Finalmente, la última forma en que se distribuye el Fondo Social Minero es para contribuir en la estrategia de Desarrollo Institucional (figura 3); en la que se realizan proyectos para empoderar a las instituciones locales, además las comunidades asumen una participación activa en los temas que atañen a la localidad. Finalmente, se generan alianzas entre líderes comunitarios, empresas, estado y ONG.

\section{Figura 2. Estrategia de reducción de la pobreza}

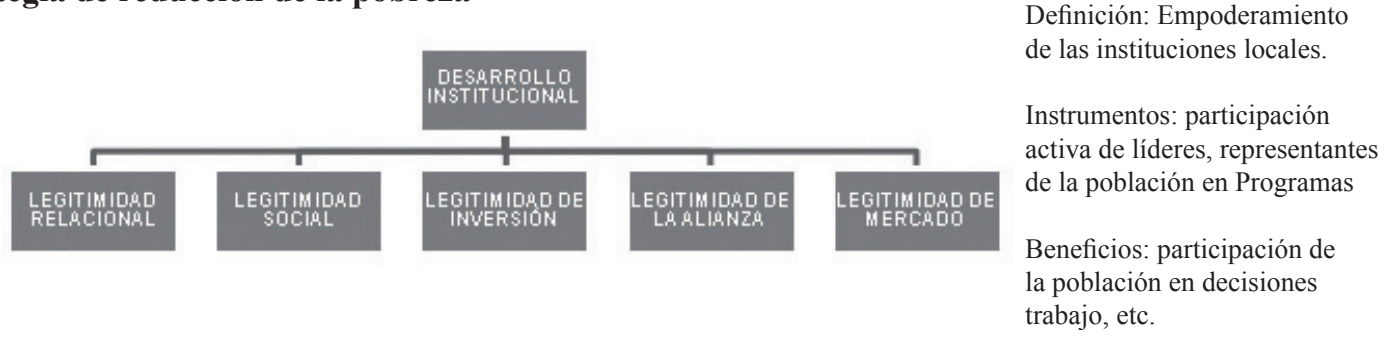

Fuente. Autora.

Pensamos que en el caso de la gran minería peruana, este modelo resultaría un aporte fundamental en la medida que se puede determinar no sólo la cantidad de alianzas establecidas sino además analizar la legitimidad que se persigue por cada alianza. Esto resulta una estrategia relevante en la administración de los recursos del Fondo Social Minero, pues de esta manera, se puede monitorear eficazmente cada alianza estratégica y fomentar o generar alianzas que se observen que benefician en mayor medida al país.

En ese sentido, el sector privado tiene un enorme potencial para generar el crecimiento al brindar un fondo que fomenta a su vez la participación activa del estado y de 
otros actores sociales. Así, la minería en el Perú resulta ser una importante fuente de desarrollo pero sólo si es gestionada a través de alianzas estratégicas y favoreciendo el cumplimiento de los objetivos de desarrollo sostenible, y reducción de la pobreza.

Son estas alianzas las que van a gestionar que el fondo minero sea empleado para ejecutar proyectos de desarrollo tomando en cuenta la armonía de las comunidades y sin

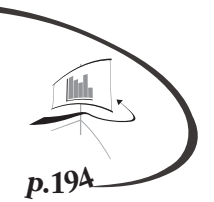
afectar el medio ambiente. Así mismo, servirán para mejorar la calidad de vida de los pobladores ayudando a reducir los conflictos que pudieran surgir debido a la inversión minera; así como para potenciar

las instituciones locales aliándose con los representantes de la zona.

Ahora, se ha visto cómo las alianzas pueden generar desarrollo desde diferentes perspectivas, sin embargo, resulta imperativo considerar la perspectiva de la Interculturalidad. Es decir, la línea que cruza este modelo es el proceso de interacción entre las diversas culturas que en el camino se van estableciendo.

En ese sentido, se ha señalado que el fondo minero es un instrumento ubicado como generador de desarrollo si es bien administrado por las instituciones pertinentes. Asimismo, este proceso genera a su vez la formación de alianzas entre las diversas partes interesadas ya sea en las tres estrategias propuestas, desarrollo sostenible, reducción de pobreza y desarrollo institucional. Este instrumento a su vez permite acompañar todo el proceso de negociación entre las diferentes culturas implicadas en la alianza.

En otras palabras, las alianzas, legitimadas, ya sea por cualquiera de las legitimidades propuestas por Dacin son un facilitador poderoso en los conflictos que pudieran surgir en el intercambio cultural. En el caso del sector minero, se ha constatado que efectivamente, existen por un lado expectativas a corto plazo de las transnacionales que invierten en la minería y por el otro lado metas a largo plazo de las comunidades de la zona. De modo que, los conflictos en la zona ocurren no sólo por la divergencia de intereses económicos, sino además por una dimensión cultural que se relaciona con la identidad, la percepción del mundo, de la naturaleza, etc. De ahí que, se establece un conflicto entre una cultura propiamente occidentalizada que tiene una percepción del trabajo, del medio ambiente y del tiempo distinta de la percepción propiamente de la visión de la comunidad (CIES, 2007).

El tema del uso de los recursos naturales, por ejemplo, es un factor central en los conflictos entre las comunidades y las empresas mineras. De un lado, para la cultura occidental, la naturaleza es observada como explotable, mensurable, tiene una valoración de uso y de aporte económico. Desde la visión andina, la naturaleza está interrelacionada en su totalidad, sus elementos como el suelo, el agua, el tiempo, están estrechamente interconectados (CIES, 2007).

Asimismo, para las comunidades, el riesgo de invertir en minería conlleva una asegurada contaminación del medio ambiente, por lo que se resisten a dar cabida a este tipo de actividad. Por el contrario, las empresas mineras se jactan de saber argumentar y de saber planificar estos riesgos que aparentemente no los perciben.

Este contexto hace imperativo que se planteen soluciones, de ahí que las alianzas estratégicas devienen como elemento de negociación cultural. Romero (2002) propone etapas que describen el proceso de formación de una alianza y que son adecuadas retomarlas en el caso del sector minero para seguir un proceso legítimo y válido tanto para las empresas, como para las comunidades en cuestión.

Entre las etapas se encuentran en primer lugar el establecimiento de la misión, los objetivos y las estrategias de cada una de las partes implicadas, luego se establecen los socios que participarán, para iniciar la etapa de negociaciones en la que se declaran ciertos principios que regirán el acuerdo. Finalmente se comienza la planificación de operaciones al detalle. Se estructura la alianza legalmente y determinando las funciones de sus partes.

Ahora, parece que el punto en el que las partes se detienen a negociar resulta el más importante pues es ahí donde se expresarán las diferencias culturales, de valores, de opinión, de percepción, etc. En el caso de la minería conviene prestar mucha más atención pues es precisamente donde devienen posibles conflictos entre dos maneras de ver el mundo, la occidental y la andina.

Siguiendo estos principios para la negociación se dice que la alianza se convertirá en un facilitador sólo si se cumple con ciertos principios tales como: que las partes ganen por 
mutuo acuerdo, que exista un compromiso serio, que se emplee la persuasión, dando pero también recibiendo, no delegación, es decir, los responsables son los que tienen que estar encabezando la alianza. Asimismo, deben de darse la comunicación intercultural, siendo flexibles y aplicando conceptos de equidad, justicia y transparencia.
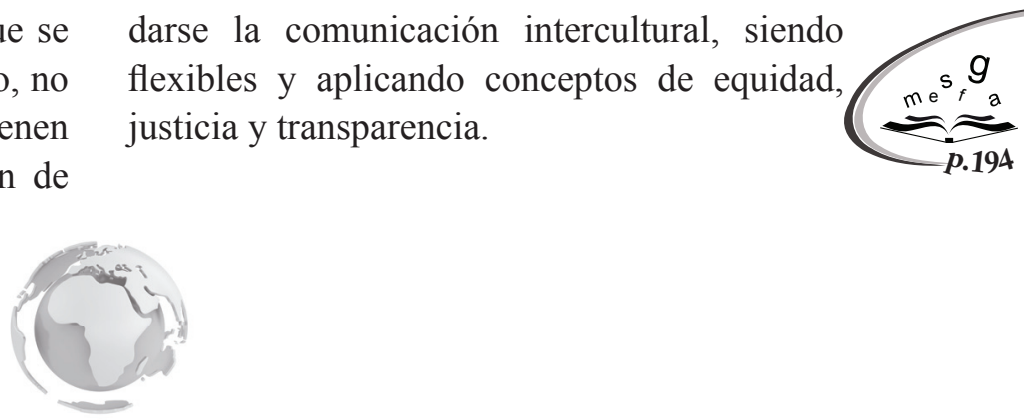

\section{CONCLUSIONES}

rente al aumento de los niveles de pobreza, se han empezado a desarrollar programas destinados al desarrollo de las comunidades más pobres, en ese sentido, el Fondo Minero resulta ser una fuente estratégica relevante para contribuir al desarrollo sostenible, la reducción de la pobreza y para el desarrollo institucional.

De otro lado, estas tres maneras de distribuir el Fondo Social Minero generan a su vez la formación de Alianzas Estratégicas que promueven programas de desarrollo. Sin embargo, para que la gestión de estas alianzas sea efectiva, es esencial que se realice un proceso de legitimización o validación en el mercado. De ahí que, se proponga un modelo integrado que permita que la gestión del Fondo
Social tenga un mejor desempeño. Este modelo señala que el fondo está destinado para favorecer las estrategias mencionadas y a su vez las alianzas que se generen pueden tener necesidades de legitimación de cinco tipos. Finalmente este modelo contribuye en el mejoramiento de la gestión del Fondo Minero y además abre nuevas perspectivas para aplicar este modelo a otros ámbitos de crecimiento. Sin embargo, se debe además tomar en cuenta las diferencias entre culturas que desde la perspectiva de la interculturalidad resultan esenciales en el proceso de negociación. Sólo si este tema es considerado se podrá hablar de alianzas como facilitadores entre culturas y como instrumento de desarrollo.

\section{REFERENCIAS BIBLIOGRÁFICAS}

Adler, N. (1991). International Dimensions of Organizational Behavior. 1st ed. Boston, MA: PWS-Kent Publishing Company.

Allan, J, J. (2001). Public-Private Partnerships: A Review of Literatura and Practice. Saskatchewan Institute of Public Policy-Public Policy Paper No4.

Aldrich, H. E. and Fiol, C. M. (1994). Fools rush in? The institutional context of industry creation. Academy of Management Review, 19, 645-70.

Arbaiza, L. (2008). La inversión privada en Responsabilidad Social Empresarial. Lima: Universidad ESAN.

Casado Cañeque, F. (2007). Alianzas Público Privadas para el Desarrollo, Documento de Trabajo N9, Fundación Carolina CeALCI, Madrid, http://www.fundacioncarolina.es

Banco Interamericano de Desarrollo (2003). Reducción de la pobreza y promoción de la equidad social.

Banco Mundial (2002). World Development Report 2003: Sustainable Development in a Dynamic World. Transforming Institutions, Growth \& Quality of Life, Nueva Cork: Oxford University Press. 
Baum O. (1991) Institutional linkages and organizational mortality. Administrative Science Quarterly 36: 187-218.

CEDEPAS (2009). Fondo Minero de Solidaridad-Cajamarca. Boletín de Vigilancia N ${ }^{\circ} 3$.

Cichon, M et al, (2003). Red del Fideicomiso Social Mundial: Nueva herramienta para combatir la pobreza mediante la protección social. OIT.

Das, T. K. and Teng, B. (2000). Instabilities of strategic alliances: an internal tensions perspective. Organization Science, $11,77-101$.

Chappuis, J. (2007) Fidecomiso privado, un ejemplo a seguir. Revista Horizonte Minero. Lima..

García-Canal et al., (2004). El papel de las alianzas estratégicas en la internacionalización de las empresas españolasUniversia Business Review,

Dacin, M. et al, (2007). The Legitimacy of Strategic Alliances: An Institutional Perspective Strategic Management Journal, 28: 169-187.

Dyer, J. H., Kale, P. y Singh, H. (2001) How to Make Strategic Alliance Work. MIT Sloan Management Review, Volumen 42, Número 4.

Fiszbein, A. \& Lowden, P. (1999). Trabajando Unidos para el Cambio: Alianzas Público-Privadas para la Reducción de la Pobreza en América Latina y el Caribe. Banco Mundial. Washington, D. C.

Francke P. \& Iguiñiz, J. (2006) Crecimiento con inclusión en el Perú. Pontificia Universidad Católica del Perú. Lima.

Gulati R. (1998). Alliances and networks. Strategic Management Journal, Special Issue 19: 293-317.

Gulati, R., Khanna,T. y Nohria, N. (1994). Unilateral Commitments and the Importance of Process in Alliances.

Sloan Management Review, Spring, págs. 61-69.

Guzmán, A. (2003). La Minería y el Desarrollo Sostenible. Medio Empresarial, año VI, N ${ }^{\circ} 54$.

Horizonte Minero. Desarrollo para las regiones. Septiembre, 2009.

Hofstede, G. (1997) Cultures and organizations: Software of the mind, New York: McGraw-Hill,

Human, S. E. and Provan, K. G. (2000). Legitimacy building in the evolution of small-firm multilateral networks: a comparative study of success and demise'. Administrative Science Quarterly, 45, 327-65.

Inforesources. Desarrollo rural a través de las Alianzas Público-Privadas (APP). Focus $\mathrm{n}^{\circ} 1 / 05$

Ireland, R., Hitt, M., \& Vaidyanath, D. (2002). Alliance Management as a Source of Competitive Advantage. Journal of Management, 28(3), 413-446. Retrieved from Business Source Complete database

Irrmann, O. \& Arcand, S. (2008). La administración intercultural fuente: extraído de http://zonecours.hec.ca/documents/ A2008-1-1660835.Irrman-Arcand11.pdf, abril 2011

Jorgensen, s. et al, (1999). The role of social funds. Inter-American Development Bank. Conference on social protection and poverty. IDB.

Larson A. (1992). Network dyads in entrepreneurial settings: a study of the governance of Exchange relationships. Administrative Science Quarterly 37: 76-104 
Lorange, R., J. y Simcic B. (1992). Building Successful Strategic Alliances. Long Range Planning, 25 (6),10-17.

Maldonado, K. (2006). Aproximación a un marco conceptual y teórico sobre la Interculturalidad de los negocios internacionales. Documentos de Investigación. Universidad del Rosario.

Minería (2009). Fondos Sociales para la Sostenibilidad. № 384.

Montes, A. \& Sabater, R. (2002). Alianzas Estratégicas: Modelo para la Gestión del proceso de negociación. Revista de Empresa $N^{\circ} 2$.extraído el 15 de junio de www.revistadeempresa.com.

Naciones Unidas (2000). Declaración del Milenio. Nueva York (2005): Documento Final de la Cumbre Mundial. A/ RES/60/1. Nueva York.

Navarro, F. (2009). La Minería seguirá contribuyendo al crecimiento del país. Horizonte Minero PE (63).

Negocios Internacionales (2009). Fondo Minero Antamina, contribuyendo al desarrollo sostenible de Ancash. $\mathrm{N}^{\circ} 141$, vol 12.

Organización Internacional del Trabajo. Programa Modular de Capacitación e Información sobre Género, Pobreza y Empleo. OIT. 2001

Parker, A. \& Serrano, R. (2000). Promoting Good Local Governance through Social Funds and Decentralization. World Bank. Washington.

PROINVERSION. Las Bambas Un Modelo de Desarrollo sostenible. Lima. 2005.

Rajesh K. \& T. K. Das (2007). Interpartner Legitimacy in the Alliance, Development Process. Journal of Management Studies 44:8.

Reardon, K.K. y Spekman, R.E. (1994). “Starting Out Right: Negotiation Lessons for Domestic and Cross-Cultural Business Alliances”. Business Horizons, January-February, 71-80.

Ruef, M. and Scott, W. R. (1998). A multidimensional model of organizational legitimacy: hospital survival in changing institutional environments'. Administrative Science Quarterly, 43, 877-904.

Sociedad Nacional de Minería Petróleo y Energía (2010). Programa Minero de Solidaridad con el Pueblo-Aporte Voluntario. Informe quincenal de la snmpe.

Suchman, M. C. (1995). Managing legitimacy: strategic and institutional approaches. Academy of Management Review, 20, 571-610.

Smith, P.R. y Van de Ven, A.H. (1994). Developmental Processes of Cooperative Interorganizational Relationships. Academy of Management Review, 19 (1), págs. 90-118.

Tendler.J. (2000). Why are social funds so popular?. Local Dynamics in an Era of Globalization. World Bank.

Tennyson, R. (2003). Manual de trabajo en Alianza en: http://www.iblf.org/docs/PartneringToolbookEspanol.pdf

WORLD BANK. Social Funds Assessing Efectiveness. World Bank. 2002

Zarco-Jasso, H. (2005) Pubic-private Partnerships: A Multidimensional Model for Contracting. IESE Business School, Universidad de Navarra, Barcelona. 